\title{
Sivers asymmetry in the photoproduction of a $J / \psi$ and a jet at the EIC
}

\author{
Raj Kishore® and Asmita Mukherjee® \\ Department of Physics, Indian Institute of Technology Bombay, Powai, Mumbai 400076, India \\ Sangem Rajesh \\ Dipartimento di Fisica, Università di Cagliari, Cittadella Universitaria, I-09042 Monserrato (CA), Italy \\ and INFN, Sezione di Cagliari, Cittadella Universitaria, I-09042 Monserrato (CA), Italy
}

(Received 19 August 2019; accepted 13 February 2020; published 4 March 2020)

\begin{abstract}
We calculate the Sivers asymmetry in the photoproduction of almost back-to-back $J / \psi$-jet pair in the process $e p^{\uparrow} \rightarrow J / \psi+$ jet $+X$, which will be possible at the future planned Electron-Ion Collider (EIC). We use the framework of a generalized parton model and nonrelativistic QCD for calculating the $J / \psi$ production rate. We include contributions from both color singlet and color octet states in the asymmetry. We obtain sizable Sivers asymmetry that can be promising to determine the gluon Sivers function. We also investigate the effect of transverse momentum dependent evolution on the asymmetry.
\end{abstract}

DOI: 10.1103/PhysRevD.101.054003

\section{INTRODUCTION}

Single spin asymmetries and transverse momentum dependent parton distributions (TMD PDFs) are objects of a lot of interest in recent days in hadron physics. Among the TMD PDFs, the Sivers function [1] is of particular interest. This gives the distribution of unpolarized quarks/ gluons in a transversely polarized nucleon, which is not left-right symmetric with respect to the plane formed by the transverse momentum and spin of the nucleon. In some model calculations the Sivers function is shown to be related to the quark orbital angular momentum through the GPD $E_{q}$ [2]. The Sivers function introduces an asymmetry, for example, in the azimuthal angle of the observed final state hadron in semi-inclusive deep inelastic scattering (SIDIS) and in the azimuthal angle correlations of the lepton pair in Drell-Yan process or back-to-back jets in $p p$ collision, called the Sivers asymmetry. The first transverse moment of the Sivers function is related to the twist-three Qiu-Sterman function [3]. First experimental information on a nonzero Sivers function for quarks was obtained from HERMES [4] and COMPASS [5] results. Since then, quite a lot of advances have been made, both in theory and experiment. Parametrization of a quark Sivers function has been obtained in [6] and for gluons in [7,8] by fitting data from RHIC within the Dokshitzer-Gribov-LipatovAltarelli-Parisi (DGLAP) evolution approach. TMDs

Published by the American Physical Society under the terms of the Creative Commons Attribution 4.0 International license. Further distribution of this work must maintain attribution to the author(s) and the published article's title, journal citation, and DOI. Funded by SCOAP ${ }^{3}$. evolve with scale in a different way compared to the collinear PDFs. Much progress has been made in the past few years to understand the TMD evolution [9-11], and unpolarized distributions and fragmentation function have been calculated at next-to-next-to-leading order [12]. A parametrization of the Sivers function incorporating the TMD evolution has been obtained in [13]; however, gluon Sivers function (GSF) is not yet known. Therefore, compared to the quark TMDs, gluon TMDs are much less known, and these will also be investigated at the future Electron-Ion Collider (EIC) [14] and the future fixed target plans at the LHC [15-17]. Gluon TMDs satisfy the positivity bounds first derived in [18]. A phenomenological bound on GSF was obtained [19], commonly known as Burkardt sum rule, from the requirement that the net transverse momentum of all partons (quarks and gluons) in a transversely polarized nucleon should vanish. In [20], a fit to the data from SIDIS at low scale indicates that this sum rule is almost saturated by contribution from $u$ and $d$ quarks; however, there may still be about $30 \%$ contribution from GSF.

The Sivers function is a T-odd object and initial and final state interactions play an important role in Sivers asymmetry [21]. They are resummed into the gauge link or Wilson line in the operator definition of the Sivers function that is needed for color gauge invariance [22]. Gluon TMDs have two gauge links, in contrast to quark TMDs, that have only one. This introduces process dependence in them. The Sivers function in SIDIS is expected to be equal in magnitude but opposite in sign compared to the Sivers function appearing in the Drell-Yan process [21]. Recent data from RHIC [23] as well as COMPASS [24] seem to favor the sign change; however, more data are needed [6]. The GSF for any process, in general, can be written in terms 
of two independent functions; one of them has an operator structure that is C-even, and the other, C-odd. In the literature, the former is called an f-type Sivers function and the latter, d-type $[25,26]$. In fact, one of these two (d-type) is not constrained by the Burkardt sum rule. More experimental data are needed to precisely determine the GSF. As is well known, $J / \psi$ production in $e p$ and $p p$ collision is an effective method to probe the gluon TMDs including the GSF [27-33], as a contribution to the Sivers asymmetry comes already at leading order (LO) through the virtual photon-gluon or gluon-gluon fusion processes, respectively. Data on Sivers asymmetry in $J / \psi$ production are available from the COMPASS Collaboration [34]; although with large error bars, it can be qualitatively explained by a LO calculation in nonrelativistic QCD (NRQCD) based color octet model [30]. In a recent work [35], maximal values of the azimuthal asymmetries in back-to-back electroproduction of a $J / \psi$ and a jet is estimated within the TMD factorization framework by neglecting the intrinsic transverse momentum of the initial parton in the hard part. Another interesting process to probe the GSF is quasireal photoproduction of a hadron [36] or $J / \psi[29,37,38]$. Contribution to the single spin asymmetry (SSA) comes from a $J / \psi$ observed in the forward region, that is when the transverse momentum $\left(p_{T}\right)$ of $J / \psi$ is small.

In this work, we investigate the possibility to probe the GSF in quasireal photoproduction of back-to-back $J / \psi$ and a jet by employing the generalized parton model (GPM) wherein the intrinsic transverse momentum of the initial parton is considered in the hard part, which will be possible in the future EIC. This will be sensitive to the GSF in a different kinematical region, and the $J / \psi$ observed need not be in the forward region.

As mentioned above, initial and final state interactions play a very important role in the SSAs. TMD factorization has been proven only for certain processes. The current status of the TMD factorization for heavy quarkonium production in $p p$ collisions can be found in [39]. The most widely used approach to calculate amplitudes for $J / \psi$ production is based on nonrelativistic QCD. In this approach, the amplitude is factorized into a soft nonperturbative part and a hard part [40-46]. The heavy quark pair is produced in color singlet (CS) or color octet (CO) states in hard interaction. This is calculated in the perturbation theory. Then this heavy quark pair hadronizes to a quarkonium by emitting soft gluons. The hadronization process is described in terms of long distance matrix elements (LDMEs), that are obtained by fitting experimental data. The LDMEs have definite scaling properties with respect to the velocity parameter $v$, which is assumed to be small $v \ll 1$ [47]. The theoretical estimates are arranged in a double expansion in powers of $v$ and the strong coupling, $\alpha_{s}$. NRQCD has been successful in explaining hadroproduction data from TEVATRON [48,49] and also $J / \psi$ photoproduction data from HERA [50-53]. Both CS and
CO contributions are needed to explain the HERA data [29]. In this work we calculate the weighted Sivers asymmetry, $A_{N}^{\sin \left(\phi_{q}\right)}$, in photoproduction of back-to-back $J / \psi$ and a jet at EIC in NRQCD by incorporating both CS and CO states. The plan of the paper is as follows. In Sec. II we give the analytic expressions of the asymmetry. In Sec. III we present the TMD evolution framework. Numerical results and conclusion are given in Secs. IV and $\mathrm{V}$, respectively.

\section{SIVERS ASYMMETRY}

We consider the photoproduction process

$$
e(l)+p^{\uparrow}(P) \rightarrow J / \psi\left(P_{\psi}\right)+\operatorname{jet}\left(P_{j}\right)+X,
$$

where the arrow in the superscript indicates the polarization of the proton. The letters in the round brackets represent the four momentum of the corresponding particles. We consider the proton-electron center-of-mass frame wherein the proton and electron move along the $+z$ and $-z$ direction. The transverse plane, defined in Fig. 1, is orthogonal to the momentum of proton direction. The initial scattering electron radiates the virtual photon that will interact with the proton. The four momentum square of the virtual photon is $q^{2} \approx-2 E_{e} E_{e}^{\prime}(1-\cos \theta)$, and $E_{e}$ and $E_{e}^{\prime}$ are energies of the initial and final scattered electron, respectively. In the forward scattering limit, photoproduction, the four momentum of the virtual photon $q^{2}=-Q^{2} \rightarrow 0$ as a result the virtual photon becomes the real photon. The dominant subprocess for $J / \psi$ production is the $\gamma(q)+$ $g(p) \rightarrow J / \psi\left(P_{\psi}\right)+g\left(P_{j}\right)$ at next-to-leading order (NLO) in $\alpha_{s}$. The quark (antiquark) initiated subprocess can also contribute $\gamma+q(\bar{q}) \rightarrow J / \psi+q(\bar{q})$. The unpolarized differential cross section for the $e p \rightarrow J / \psi+$ jet $+X$ process can be written as follows:

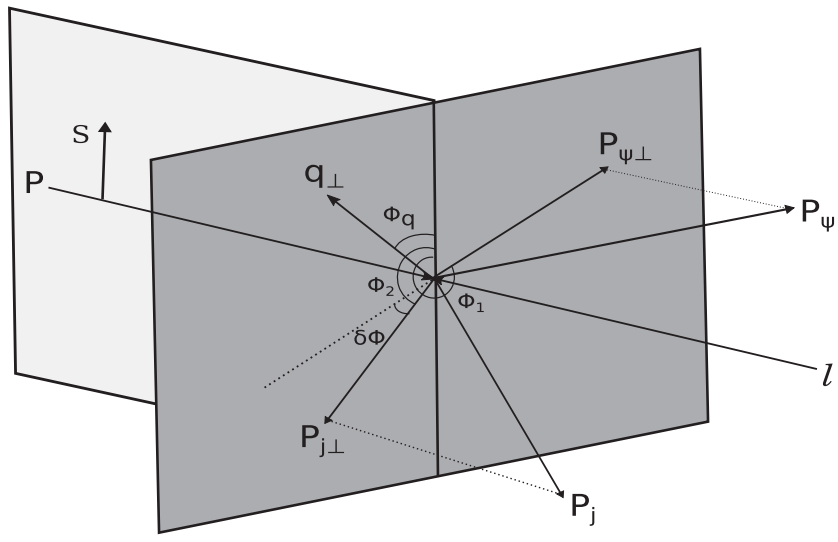

FIG. 1. Illustration of azimuthal angles in the $e p^{\uparrow} \rightarrow J / \psi+$ jet process. The transverse momenta $\boldsymbol{P}_{\Psi \perp}$ and $\boldsymbol{P}_{j \perp}$ of a $J / \psi$ and a jet, respectively, are in the plane orthogonal to the momentum of the proton $P$. 


$$
\begin{aligned}
E_{\Psi} E_{j} \frac{d \sigma}{d^{3} \boldsymbol{P}_{\Psi} d^{3} \boldsymbol{P}_{j}}= & \frac{d \sigma}{d^{2} \boldsymbol{P}_{\Psi \perp} d z d^{2} \boldsymbol{P}_{j \perp} d z_{1}} \\
= & \frac{1}{4(2 \pi)^{2}} \frac{1}{z z_{1}} \sum_{a} \int d x_{\gamma} d x_{a} d^{2} \boldsymbol{p}_{a \perp} f_{\gamma / e}\left(x_{\gamma}\right) \\
& \times f_{a / p}\left(x_{a}, p_{a \perp}\right) \\
& \times \delta^{4}\left(q+p-P_{\Psi}-P_{j}\right) \frac{1}{2 \hat{s}}\left|\mathcal{M}_{\gamma a \rightarrow J / \psi a}\right|^{2}
\end{aligned}
$$

where $a=g, u, d, s, \bar{u}, \bar{d}, \bar{s}$. The $x_{\gamma}$ and $x_{a}$ are the lightcone momentum fractions of photon and partons, respectively, and $\boldsymbol{p}_{a \perp}$ is the transverse momentum of the initial parton. We have assumed TMD factorization in the GPM model with the inclusion of intrinsic transverse momentum of the initial parton in the hard part. When the exchanged photon is quasireal, the inreaction takes place through the Weizsäker-Williams distribution function of the electron, $f_{\gamma / e}\left(x_{\gamma}\right)$; this describes the density of photons inside the electron and is given by [54]

$$
\begin{aligned}
f_{\gamma / e}\left(x_{\gamma}\right)= & \frac{\alpha}{2 \pi}\left[2 m_{e}^{2} x_{\gamma}\left(\frac{1}{Q_{\min }^{2}}-\frac{1}{Q_{\max }^{2}}\right)\right. \\
& \left.+\frac{1+\left(1-x_{\gamma}\right)^{2}}{x_{\gamma}} \ln \frac{Q_{\max }^{2}}{Q_{\min }^{2}}\right],
\end{aligned}
$$

where $\alpha$ is the electromagnetic coupling and $Q_{\min }^{2}=m_{e}^{2} \frac{x_{\gamma}^{2}}{1-x_{\gamma}}$, $m_{e}$ being the electron mass. We have considered $Q_{\max }^{2}=$ $1 \mathrm{GeV}^{2}$ for estimating the Sivers asymmetry. The unpolarized TMD, $f_{a / p}$, represents the density of unpolarized partons inside an unpolarized proton with momentum fraction $x_{a}$ and transverse momentum $p_{a \perp}$. The $\hat{s}, \hat{t}$, and $\hat{u}$ are the Mandelstam variables at partonic level and their definitions are given in Appendix A. $\mathcal{M}_{\gamma a \rightarrow J / \psi a}$ is the amplitude of gluon and quark (antiquark) initiated subprocesses. The square of the amplitude is calculated using the NRQCD model; for more details Ref. [29] is referred for gluon channel, and the quark (antiquark) channel matrix elements are given in Appendix B. The CS and CO states i.e., ${ }^{3} S_{1}^{(1,8)},{ }^{1} S_{0}^{(8)}$ and ${ }^{3} P_{J}^{(8)}$, are considered for $J / \psi$ production. The center-of-mass energy of the proton-electron system is $s=(P+l)^{2}$. In Eq. (2), the inelastic variables $z=\frac{P \cdot P_{h}}{P \cdot q}$ and $z_{1}=\frac{P \cdot P_{j}}{P \cdot q}$ are the energy fractions transferred from photon to $J / \psi$ and jet, respectively, in the proton rest frame. In photoproduction, the inelastic variables $z$ and $z_{1}$ can be measured in experiments using the Jacquet-Blondel method [50,51,53]. By using the definitions of four momenta as given in Appendix A, the momentum conservation delta function can be decomposed as

$$
\begin{aligned}
\delta^{4}\left(q+p-P_{\Psi}-P_{j}\right) \\
=\frac{2}{x_{\gamma} s} \delta\left(1-z-z_{1}\right) \delta\left(x_{a}-\frac{M^{2}+P_{\Psi \perp}^{2}}{z x_{\gamma} s}-\frac{P_{j \perp}^{2}}{z_{1} x_{\gamma} s}\right) \\
\quad \times \delta^{2}\left(\boldsymbol{p}_{a \perp}-\boldsymbol{P}_{\Psi \perp}-\boldsymbol{P}_{j \perp}\right),
\end{aligned}
$$

where $\boldsymbol{P}_{\Psi \perp}$ and $\boldsymbol{P}_{j \perp}$ are the transverse momentum of the $J / \psi$ and jet, respectively, and their azimuthal angles are represented with $\phi_{1}$ and $\phi_{2}$ such that $\delta \phi=\phi_{2}-\phi_{1}-\pi$ as shown in Fig. 1.

Now, we define the sum and difference of transverse momenta of the $J / \psi$ and jet as $\boldsymbol{q}_{\perp}=\boldsymbol{P}_{\Psi \perp}+\boldsymbol{P}_{j \perp}$ and $\boldsymbol{K}_{\perp}=\left(\boldsymbol{P}_{\Psi \perp}-\boldsymbol{P}_{j \perp}\right) / 2$. We are interested in the case where $\left|\boldsymbol{q}_{\perp}\right| \ll\left|\boldsymbol{K}_{\perp}\right|$ i.e., the $J / \psi$ and jet are almost back to back in the transverse plane as shown in Fig. 1. The azimuthal angle of $\boldsymbol{q}_{\perp}$ is denoted with $\phi_{q}$. After integrating over $z_{1}$, $x_{a}$, and $\boldsymbol{p}_{a \perp}$, one obtains the following expression:

$$
\frac{d \sigma}{d^{2} \boldsymbol{q}_{\perp} d z d^{2} \boldsymbol{K}_{\perp}}=\frac{1}{2(2 \pi)^{2}} \frac{1}{z(1-z) s} \sum_{a} \int \frac{d x_{\gamma}}{x_{\gamma}} f_{\gamma / e}\left(x_{\gamma}\right) f_{a / p}\left(x_{a}, q_{\perp}\right) \frac{1}{2 \hat{s}}\left|\mathcal{M}_{\gamma a \rightarrow J / \psi a}\right|^{2}
$$

For a tranversly polarized proton, the differential cross section is given by

$$
\frac{d \sigma^{\uparrow(\downarrow)}}{d^{2} \boldsymbol{q}_{\perp} d z d^{2} \boldsymbol{K}_{\perp}}=\frac{1}{2(2 \pi)^{2}} \frac{1}{z(1-z) s} \sum_{a} \int \frac{d x_{\gamma}}{x_{\gamma}} f_{\gamma / e}\left(x_{\gamma}\right) f_{a / p^{\uparrow(\downarrow)}}\left(x_{a}, q_{\perp}\right) \frac{1}{2 \hat{s}}\left|\mathcal{M}_{\gamma a \rightarrow J / \psi a}\right|^{2} .
$$

The weighted Sivers asymmetry is defined as [55]

$$
A_{N}^{W\left(\phi_{q}\right)} \equiv \frac{\int d \phi_{q} W\left(\phi_{q}\right)\left(\mathrm{d} \sigma^{\uparrow}-\mathrm{d} \sigma^{\downarrow}\right)}{\int d \phi_{q}\left(\mathrm{~d} \sigma^{\uparrow}+\mathrm{d} \sigma^{\downarrow}\right)} \equiv \frac{\int d \phi_{q} W\left(\phi_{q}\right) \mathrm{d} \Delta \sigma\left(\phi_{q}\right)}{\int d \phi_{q} 2 \mathrm{~d} \sigma}
$$

where $\mathrm{d} \sigma^{\uparrow(\downarrow)}$ indicates the polarized cross section in the process where one of the initial particles is transversely polarized with respect to its momentum direction. The azimuthal weight factor $W\left(\phi_{q}\right)=-\sin \left(\phi_{q}\right)$ is given by $[56,57]$

$$
-\sin \left(\phi_{q}\right)=\frac{(\boldsymbol{S} \times \hat{\boldsymbol{P}}) \cdot \boldsymbol{q}_{\perp}}{|\boldsymbol{S} \times \hat{\boldsymbol{P}}|\left|\hat{\boldsymbol{P}} \times \boldsymbol{q}_{\perp}\right|} .
$$

It has been advertised that the $J / \psi$ production probes the gluon TMDs and the quark contribution can be safely 
neglected in the kinematical region considered because of the insignificant contribution of quarks with respect to the gluon, which will be discussed in the results section. Hence the dominant contribution to the Sivers asymmetry comes from the gluon channel. The numerator of the asymmetry is sensitive to the Sivers function in $J / \psi$ production

$$
\begin{aligned}
\mathrm{d} \Delta \sigma \equiv & \frac{d \sigma^{\uparrow}}{d^{2} \boldsymbol{q}_{\perp} d z d^{2} \boldsymbol{K}_{\perp}}-\frac{d \sigma^{\downarrow}}{d^{2} \boldsymbol{q}_{\perp} d z d^{2} \boldsymbol{K}_{\perp}} \\
= & \frac{1}{2(2 \pi)^{2}} \frac{1}{z(1-z) s} \sum_{a} \int \frac{d x_{\gamma}}{x_{\gamma}} f_{\gamma / e}\left(x_{\gamma}\right) \Delta \hat{f}_{a / p}\left(x_{a}, \boldsymbol{q}_{\perp}\right) \\
& \times \frac{1}{2 \hat{s}}\left|\mathcal{M}_{\gamma a \rightarrow J / \psi a}\right|^{2},
\end{aligned}
$$

with $\Delta \hat{f}_{a / p^{\uparrow}}\left(x_{a}, \boldsymbol{q}_{\perp}\right)$ being the Sivers function, and describes the number density of unpolarized partons in a transversely polarized proton with mass $M_{p}$. The analytic expressions for contributions from different states can be found in [29]. As only a $J / \psi$ was observed there, we had integrated over the phase space of the final gluon $a=g$, whereas here, the final parton is producing the observed jet. The Sivers function in Trento convention [56] is given by

$$
\begin{aligned}
\Delta \hat{f}_{a / p^{\uparrow}}\left(x_{a}, \boldsymbol{q}_{\perp}\right) & \equiv \hat{f}_{a / p^{\uparrow}}\left(x_{a}, \boldsymbol{q}_{\perp}\right)-\hat{f}_{a / p^{\downarrow}}\left(x_{a}, \boldsymbol{q}_{\perp}\right) \\
& =\Delta^{N} f_{a / p^{\uparrow}}\left(x_{a}, q_{\perp}\right) \hat{\boldsymbol{S}} \cdot\left(\hat{P} \times \hat{\boldsymbol{q}}_{\perp}\right) \\
& =-\Delta^{N} f_{a / p^{\uparrow}}\left(x_{a}, q_{\perp}\right) \sin \left(\boldsymbol{\phi}_{q}\right) \\
& =-\frac{2}{M_{p}} f_{1 T}^{\perp}\left(x_{a}, q_{\perp}\right) \hat{\boldsymbol{S}} \cdot\left(\hat{P} \times \boldsymbol{q}_{\perp}\right) .
\end{aligned}
$$

The Sivers function fulfills the following positivity bound:

$$
\begin{aligned}
\left|\Delta^{N} f_{a / p^{\uparrow}}\left(x_{a}, q_{\perp}\right)\right| & \leq 2 f_{a / p}\left(x_{a}, q_{\perp}\right), \quad \text { or } \\
\frac{q_{\perp}}{M_{p}}\left|f_{1 T}^{\perp}\left(x_{a}, q_{\perp}\right)\right| & \leq f_{a / p}\left(x_{a}, q_{\perp}\right) .
\end{aligned}
$$

Following Refs. [7,8], we adopt the Gaussian parametrization for the Sivers function within the DGLAP evolution approach as

\begin{tabular}{|c|c|c|c|c|c|c|c|}
\hline \multicolumn{8}{|c|}{ Best fit parameters } \\
\hline Evolution & $a$ & $N_{a}$ & $\alpha$ & $\beta$ & $\rho$ & $\begin{array}{l}\left\langle q_{\perp}^{2}\right\rangle \\
\mathrm{GeV}^{2}\end{array}$ & Notation \\
\hline \multirow[t]{3}{*}{ DGLAP } & $g[7]$ & 0.65 & 2.8 & 2.8 & 0.687 & 0.25 & SIDIS1 \\
\hline & $g[7]$ & 0.05 & 0.8 & 1.4 & 0.576 & 0.25 & SIDIS2 \\
\hline & $g[8]$ & 0.25 & 0.6 & 0.6 & 0.1 & 1.0 & SIDIS3 \\
\hline \multirow[t]{2}{*}{ TMD } & $u[13]$ & 0.106 & 1.051 & 4.857 & & 0.38 & TMD-a \\
\hline & $d[13]$ & -0.163 & 1.552 & 4.857 & & 0.38 & TMD-b \\
\hline
\end{tabular}

$$
\begin{aligned}
\Delta^{N} f_{a / p^{\uparrow}}\left(x_{a}, q_{\perp}\right) & =\left(-2 \frac{q_{\perp}}{M_{p}}\right) f_{1 T}^{\perp}\left(x_{a}, q_{\perp}\right) \\
& =2 \mathcal{N}_{a}\left(x_{a}\right) f_{a / p}\left(x_{a}\right) h\left(q_{\perp}\right) \frac{e^{-q_{\perp}^{2} /\left\langle q_{\perp}^{2}\right\rangle}}{\pi\left\langle q_{\perp}^{2}\right\rangle},
\end{aligned}
$$

where $f_{a / p}\left(x_{a}\right)$ is the usual collinear parton distribution function which follows the DGLAP evolution equation and

$$
\mathcal{N}_{a}\left(x_{a}\right)=N_{a} x_{a}^{\alpha}\left(1-x_{a}\right)^{\beta} \frac{(\alpha+\beta)^{(\alpha+\beta)}}{\alpha^{\alpha} \beta^{\beta}},
$$

with $\left|N_{a}\right| \leq 1$ and
TABLE I. Best fit parameters of Sivers function.

as a result the Sivers function satisfies the positivity bound for all values of $x_{a}$ and $q_{\perp}$. If we define the parameter

$$
\rho=\frac{M^{\prime 2}}{\left\langle q_{\perp}^{2}\right\rangle+M^{\prime 2}},
$$

such that $0<\rho<1$, then Eq. (12) becomes

$$
\begin{aligned}
& \Delta^{N} f_{a / p^{\uparrow}}\left(x_{a}, q_{\perp}\right) \\
& \quad=2 \frac{\sqrt{2 e}}{\pi} \mathcal{N}_{a}\left(x_{a}\right) f_{a / p}\left(x_{a}\right) \sqrt{\frac{1-\rho}{\rho}} q_{\perp} \frac{e^{-q_{\perp}^{2} / \rho\left\langle q_{\perp}^{2}\right\rangle}}{\left\langle q_{\perp}^{2}\right\rangle^{3 / 2}} .
\end{aligned}
$$

The unpolarized gluon TMD sitting in the denominator of the asymmetry is parametrized as Gaussian distribution

$$
f_{a / p}\left(x_{a}, q_{\perp}\right)=\frac{1}{\pi\left\langle q_{\perp}^{2}\right\rangle} f\left(x_{a}\right) e^{-q_{\perp}^{2} /\left\langle q_{\perp}^{2}\right\rangle}
$$

The best fit parameters of the Sivers function have been extracted for quarks [6] and gluons [7,8] from SIDIS and RHIC data, respectively. In Ref. [8], a new set of best fit parameters of GSF are extracted for $\left\langle q_{\perp}^{2}\right\rangle=1 \mathrm{GeV}^{2}$ and are tabulated in Table I.

\section{TMD EVOLUTION}

In DGLAP evolution, collinear PDFs evolve with only the probing scale $(\mu)$. However, in the TMD evolution approach, TMDs evolve with both the intrinsic transverse momentum $\left(p_{a \perp}\right)$ of the parton and the probing scale. The TMD evolution framework is derived in the impact parameter space $\left(b_{\perp}\right)[10]$

$$
f\left(x_{a}, b_{\perp}, \mu\right)=\int d^{2} \boldsymbol{p}_{a \perp} e^{-i \boldsymbol{b}_{\perp} \boldsymbol{p}_{a \perp}} f\left(x_{a}, p_{a \perp}, \mu\right),
$$

and in the momentum space is given by 


$$
f\left(x_{a}, p_{a \perp}, \mu\right)=\frac{1}{(2 \pi)^{2}} \int d^{2} \boldsymbol{b}_{\perp} e^{i \boldsymbol{b}_{\perp} \boldsymbol{p}_{a \perp}} f\left(x_{a}, b_{\perp}, \mu\right) .
$$

Usually, TMDs depend on two scales that are renormalization scale $(\mu)$ and auxiliary scale $(\zeta)$ [58,59]. In order to cure the light-cone (rapidity) divergences in TMD factorization, the scale $\zeta$ has been introduced. One can obtain the renormalization group and Collins-Soper equations by taking scale evolution with respect to $\mu$ and $\zeta$, respectively. The unpolarized TMD expression at a given final scale $Q_{f}=\sqrt{\zeta}=M$ is obtained by solving RG and CS equations $[13,58,59]$ and is given as

$f\left(x_{a}, b_{\perp}, Q_{f}, \zeta\right)=f\left(x_{a}, b_{\perp}, Q_{i}\right) R_{\mathrm{pert}}\left(Q_{f}, Q_{i}, b_{*}\right) R_{N P}\left(Q_{f}, b_{\perp}\right)$,

where $Q_{i}=c / b_{*}\left(b_{\perp}\right)$ is the initial scale of the TMD with $c=2 e^{-\gamma_{\epsilon}}$ and $\gamma_{\epsilon} \approx 0.577$. In line with Ref. [13], we adopt the $b_{*}$ prescription to avoid hitting the Landau pole by freezing the scale $b_{\perp}$. Here, $b_{*}\left(b_{\perp}\right)=\frac{b_{\perp}}{\sqrt{1+\left(\frac{b_{\perp}}{b_{\text {max }}}\right)^{2}}} \approx b_{\text {max }}$ when $b_{\perp} \rightarrow \infty$ and $b_{*}\left(b_{\perp}\right) \approx b_{\perp}$ when $b_{\perp} \rightarrow 0$. The $R_{\text {pert }}$ and $R_{N P}$ are the perturbative and the nonperturbative parts of the TMD, respectively, which are given as

$R_{\text {pert }}\left(Q_{f}, b_{*}\right)=\exp \left\{-\int_{c / b_{*}}^{Q_{f}} \frac{d \mu}{\mu}\left(A \log \left(\frac{Q_{f}^{2}}{\mu^{2}}\right)+B\right)\right\}$

and

$$
R_{N P}\left(Q_{f}, b_{\perp}\right)=\exp \left\{-\left[g_{1}^{\mathrm{TMD}}+\frac{g_{2}}{2} \log \frac{Q_{f}}{Q_{0}}\right] b_{\perp}^{2}\right\},
$$

where the anomalous dimensions are $A=\sum_{n=1}^{\infty}\left(\frac{\alpha_{s}(\mu)}{\pi}\right)^{n} A_{n}$ and $B=\sum_{n=1}^{\infty}\left(\frac{\alpha_{s}(\mu)}{\pi}\right)^{n} B_{n}$, and the coefficients for the gluon case are $A_{1}=C_{A}, A_{2}=\frac{1}{2} C_{F}\left(C_{A}\left(\frac{67}{18}-\frac{\pi^{2}}{6}\right)-\frac{5}{9} C_{A} N_{f}\right)$ and $B_{1}=-\frac{1}{2}\left(\frac{11}{3} C_{A}-\frac{2}{3} N_{f}\right)$, and for the quark case are $A_{1}=C_{F}, A_{2}=\frac{1}{2} C_{F}\left(C_{A}\left(\frac{67}{18}-\frac{\pi^{2}}{6}\right)-\frac{5}{9} N_{f}\right)$ and $B_{1}=-\frac{3}{2} C_{F}$ [13]. The unpolarized TMD at the initial scale can be written as

$$
\begin{aligned}
f\left(x_{a}, b_{\perp}, Q_{i}\right)= & \sum_{i=g, q} \int_{x} \frac{d \hat{x}}{\hat{x}} C_{i / a}\left(x_{a} / \hat{x}, b_{\perp}, \alpha_{s}, Q_{i}\right) f_{i / p}\left(\hat{x}, c / b_{*}\right) \\
& +\mathcal{O}\left(b_{\perp} \Lambda_{\mathrm{QCD}}\right),
\end{aligned}
$$

where $C_{i / a}$ is the perturbatively calculated process independent coefficient function, and is different for each type of TMD. The derivative of the Sivers function follows the same evolution equation as given in Eq. (20) but the $g_{1}^{\mathrm{TMD}}$ changes to $g_{1}^{\text {Sivers }}$ in the $R_{N P}$ factor. The Sivers function $f_{1 T}^{\perp}\left(x_{a}, p_{a \perp}, Q_{f}\right)$ and its derivative are related by Fourier transformation as [59]

$$
\begin{aligned}
& f_{1 T}^{\perp}\left(x_{a}, p_{a \perp}, Q_{f}\right) \\
& \quad=-\frac{1}{2 \pi p_{a \perp}} \int_{0}^{\infty} d b_{\perp} b_{\perp} J_{1}\left(p_{a \perp} b_{\perp}\right) f_{1 T}^{\prime} \frac{1}{\left(x_{a}, b_{\perp}, Q_{f}\right),}
\end{aligned}
$$

and the unpolarized TMD is given by

$$
\begin{aligned}
& f_{a / p}\left(x_{a}, p_{a \perp}, Q_{f}\right) \\
& \quad=\frac{1}{2 \pi} \int_{0}^{\infty} d b_{\perp} b_{\perp} J_{0}\left(p_{a \perp} b_{\perp}\right) f_{a / p}\left(x_{a}, b_{\perp}, Q_{f}\right) .
\end{aligned}
$$

The derivative of the Sivers function at the initial scale $Q_{i}$ can be written in terms of a Qiu-Sterman function as $[22,60]$

$$
f_{1 T}^{\prime \perp}\left(x_{a}, b_{\perp}, Q_{i}\right) \simeq \frac{M_{p} b_{\perp}}{2} T_{a, F}\left(x_{a}, x_{a}, Q_{i}\right),
$$

where $T_{a, F}\left(x_{a}, x_{a}, Q_{i}\right)$ is the Qiu-Sterman function, which is usually assumed to be proportional to collinear PDF $[13,61]$

$$
T_{a, F}\left(x_{a}, x_{a}, Q_{i}\right)=\mathcal{N}_{a}\left(x_{a}\right) f_{a / p}\left(x_{a}, Q_{i}\right) .
$$

The definition of $\mathcal{N}_{a}\left(x_{a}\right)$ is given in Eq. (13). Note that here we have used the fact that the Sivers function in a Drell-Yan process has opposite sign compared to the Sivers function in semi-inclusive DIS. So far the best fit parameters of GSF have not been extracted in the TMD evolution approach. However only the $u$ and $d$ quark Sivers function are known which were extracted in [13] from SIDIS data within the TMD evolution scheme, which are tabulated in Table I. So far a fit for the gluon Sivers function is not available in the TMD evolution approach. In order to show the effect of this evolution on the asymmetry, we use an exploratory approach; namely, following Ref. [62], we define the following two sets of parametrizations for GSF by using the known $u$ and $d$ quark Sivers function parameters

$$
\begin{aligned}
& \text { (a) } \mathcal{N}_{g}\left(x_{g}\right)=\left(\mathcal{N}_{u}\left(x_{g}\right)+\mathcal{N}_{d}\left(x_{g}\right)\right) / 2, \\
& \text { (b) } \mathcal{N}_{g}\left(x_{g}\right)=\mathcal{N}_{d}\left(x_{g}\right) .
\end{aligned}
$$

We denote the first parametrization as TMD-a and second one as TMD-b. As the sign of $N_{u}$ and $N_{d}$ are opposite, TMD-a gives a small GSF and TMD-b gives a large GSF. As mentioned in the Introduction, Burkardt sum rule [19] gives a constraint on the GSF. However, in order to implement the constraint from the Burkardt sum rule contribution, all quark favors need to be included, and the sea quark Sivers function is still not well constrained. Assuming contributions only from $u$ and $d$ quarks and gluons, we have checked that TMD-a parametrization satisfies the Burkardt sum rule, and violation is about $1 \%$; whereas the TMD-b parametrization violates the sum 
rule by about $19 \%$. The numerical values of best fit parameters are estimated [13] at $Q_{0}=\sqrt{2.4} \mathrm{GeV}, b_{\max }=$ $1.5 \mathrm{GeV}^{-1}, g_{2}=0.16 \mathrm{GeV}^{2}$, and $\left\langle p_{s \perp}^{2}\right\rangle=0.282 \mathrm{GeV}^{2}$ with $g_{1}^{\mathrm{PDF}}=\left\langle p_{a \perp}^{2}\right\rangle / 4=\left\langle q_{\perp}^{2}\right\rangle / 4$ and $g_{1}^{\text {Sivers }}=\left\langle p_{s \perp}^{2}\right\rangle / 4$. The numerator and denominator parts of Eq. (7) in TMD evolution approach can be written as

$$
\begin{gathered}
\mathrm{d} \Delta \sigma=-\frac{1}{\pi M_{p}} \frac{1}{2(2 \pi)^{2}} \frac{1}{z(1-z) s} \sum_{a} \int \frac{d x_{\gamma}}{x_{\gamma}} d b_{\perp} b_{\perp} J_{1}\left(q_{\perp} b_{\perp}\right) f_{1 T}^{\prime \perp}\left(x_{a}, b_{\perp}, Q_{f}\right) f_{\gamma / e}\left(x_{\gamma}\right) \frac{1}{2 \hat{s}}\left|\mathcal{M}_{\gamma a \rightarrow J / \psi a}\right|^{2} \sin \left(\phi_{q}\right), \\
2 d \sigma=\frac{1}{2(2 \pi)^{2} \pi} \frac{1}{z(1-z) s} \sum_{a} \int \frac{d x_{\gamma}}{x_{\gamma}} d b_{\perp} b_{\perp} J_{0}\left(q_{\perp} b_{\perp}\right) f_{a / p}\left(x_{a}, b_{\perp}, Q_{f}\right) f_{\gamma / e}\left(x_{\gamma}\right) \frac{1}{2 \hat{s}}\left|\mathcal{M}_{\gamma a \rightarrow J / \psi a}\right|^{2} .
\end{gathered}
$$

\section{NUMERICAL RESULTS}

In this section, we discuss the numerical results of Sivers asymmetry in an $e p^{\uparrow} \rightarrow J / \psi+$ jet $+X$ photoproduction process, where the proton is transversely polarized. We consider the situation $\left|\boldsymbol{q}_{\perp}\right| \ll\left|\boldsymbol{K}_{\perp}\right|$ i.e., the produced pair of a $J / \psi$ and a jet are almost back to back in the transverse plane as shown in Fig. 1. This configuration is feasible in the future proposed Electron-Ion Collider with center-ofmass energy from 20 to $150 \mathrm{GeV}$. The NLO photon-gluon fusion and quark (antiquark) initiated subprocesses, $\gamma g \rightarrow$ $J / \psi g$ and $\gamma q($ or $\bar{q}) \rightarrow J / \psi q($ or $\bar{q})$, are considered. The NRQCD model is employed for $J / \psi$ production, and the $\mathrm{CS}$ and CO states are considered for both numerator and denominator parts of Eq. (7). If we do not detect the jet in the final state then the CS state does not contribute to the asymmetry, because the initial and final state interactions between the final state parton and remnant of the proton get canceled with each other as discussed in [63]. The values of long distance matrix elements are taken from Ref. [64]. There are different sets of LDMEs in the literature and the asymmetry is found to be independent of the choice of LDME set.

There are two types of $J / \psi$ photoproduction that are resolved and direct photon contributions. The resolved photoproduction, where the photon splits into partons which subsequently interact with the partons of proton, contributes to $J / \psi$ production in the low $z$ region $(z<0.3)$. While in the direct photoproduction, the photon directly interacts electromagnetically with the partons from the proton. For direct inelastic $J / \psi$ photoproduction one has to consider $0.3<z<0.9$ as discussed in Ref. [29]. The fragmentation of a gluon and a heavy quark can also contribute to $J / \psi$ production at high transverse momentum of the $J / \psi$ [65]. The feed-down contribution from an excited state $\psi(2 S)$ and the decay of $\chi_{c}$ states contribution to $J / \psi$ are $15 \%$ [52] and $1 \%[66,67]$, respectively, but are not considered in this work. The final state parton becomes soft at $z \rightarrow 1$ which leads to infrared singularity. Therefore, to calculate the asymmetry for direct inelastic $J / \psi$ photoproduction we consider $z=0.3$. The mass of $J / \psi$ is taken to be $M=3.1 \mathrm{GeV}$. The cteq611 PDF sets are used for collinear PDFs [68].

The Sivers asymmetry is calculated at EIC for $\sqrt{s}=45$ and $100 \mathrm{GeV}$ within the DGLAP and TMD evolution approaches. In the DGLAP evolution approach, the GSF has been extracted in [7] from pion data at the RHIC for fixed Gaussian width $\left\langle q_{\perp}^{2}\right\rangle=0.25 \mathrm{GeV}^{2}$. Recently refitted, the RHIC data with a new set of GSF parameters for $\left\langle q_{\perp}^{2}\right\rangle=1 \mathrm{GeV}^{2}$ [8]. However, GSF has not been extracted yet in the TMD evolution approach. The $u$ and $d$ quark Sivers functions are extracted in Ref. [13] using the TMD evolution approach. For numerical estimation of Sivers asymmetry, the $u$ and $d$ quark Sivers function best fit parameters are used for GSF as defined in Eq. (28) within the TMD evolution approach. The best fit parameters of GSF and the quark Sivers function are tabulated in Table I. The convention of figures is as follows. The obtained Sivers asymmetry in the DGLAP approach is represented with SIDIS1, SIDIS2, and SIDIS3. TMD-a and TMD-b represent the Sivers asymmetry in the TMD evolution approach.

In Figs. 2-4, the asymmetry in the DGLAP and TMD evolution approaches as a function of $q_{\perp}$ is shown at $\sqrt{s}=$ 45 and $100 \mathrm{GeV}$ for $K_{\perp}=3 \mathrm{GeV}$, respectively, at $z=0.3$. The asymmetry is shown in the range $0 \leq q_{\perp} \leq 1 \mathrm{GeV}$ which is considered to satisfy the condition $\left|\boldsymbol{q}_{\perp}\right| \ll\left|\boldsymbol{K}_{\perp}\right|$. The value of $K_{\perp}=3 \mathrm{GeV}$ is chosen of the order of the $J / \psi$ mass. For higher values of $K_{\perp}$ and $z$ the gluon channel contribution is suppressed because the momentum fraction of the parton, $x_{a}$, depends quadratically on $K_{\perp}$ which can be seen from Eq. (4).

The maximized Sivers asymmetry, $A_{N}^{\mathrm{Max}}$, at $\sqrt{s}=$ $45 \mathrm{GeV}$ is shown in Fig. 2. Here, we saturated the Sivers function bound by adopting $\mathcal{N}_{a}=1$ and $\rho=2 / 3$ [8] in the parametrization of the Sivers function which is given in Eq. (12). In the left panel of Fig. 2, the gluon and quark (antiquark) channels contribution to $A_{N}^{\mathrm{Max}}$ is shown, and the quark (antiquark) channel contribution is insignificant compared to the gluon channel. On this basis we can say that the pair of $J / \psi+$ jet photoproduction process is an effective channel that probes the poorly known GSF in 

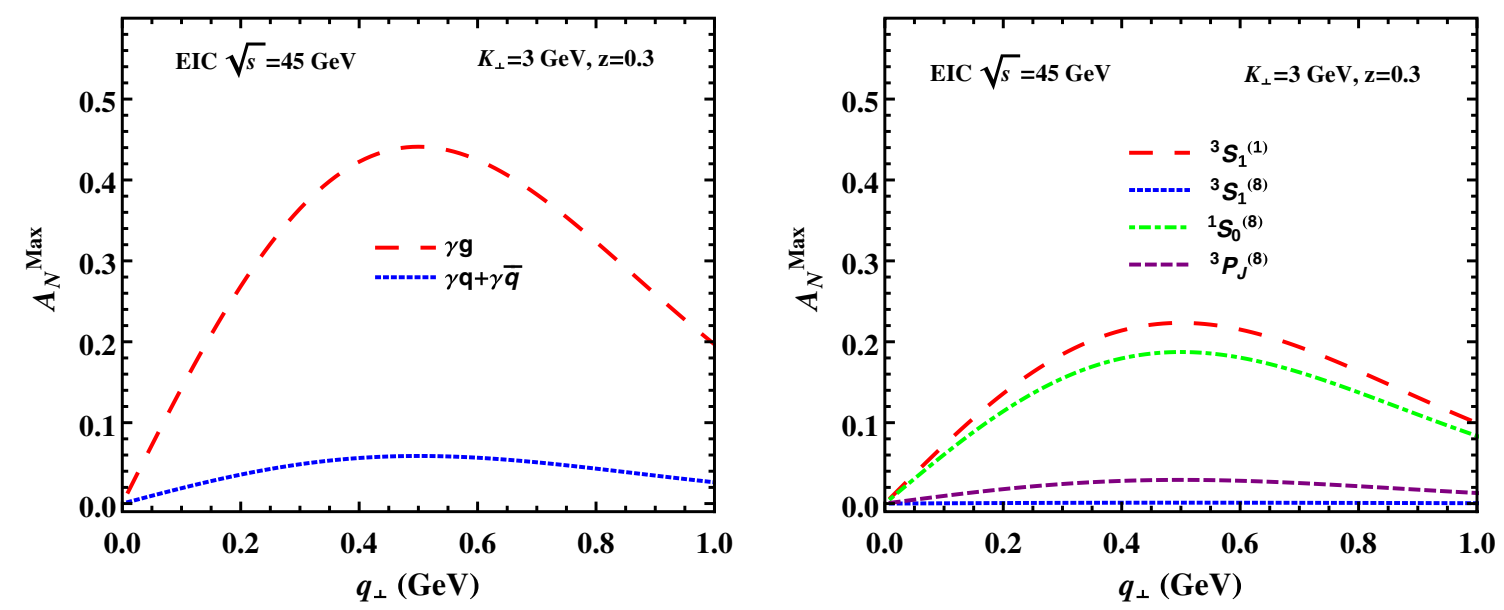

FIG. 2. Maximized Sivers asymmetry in an $e+p^{\uparrow} \rightarrow J / \psi+$ jet $+X$ process as a function of $q_{\perp}$ at EIC $\sqrt{s}=45 \mathrm{GeV}$. The Sivers function is saturated by adopting $\mathcal{N}_{g}(x)=1$ and $\rho=2 / 3$ for the parametrization of the Sivers function given in Eq. (16). Left: gluon and quark (antiquark) initiated subprocesses contribution to the asymmetry. Right: different CS and CO states contribution to the maximum asymmetry.
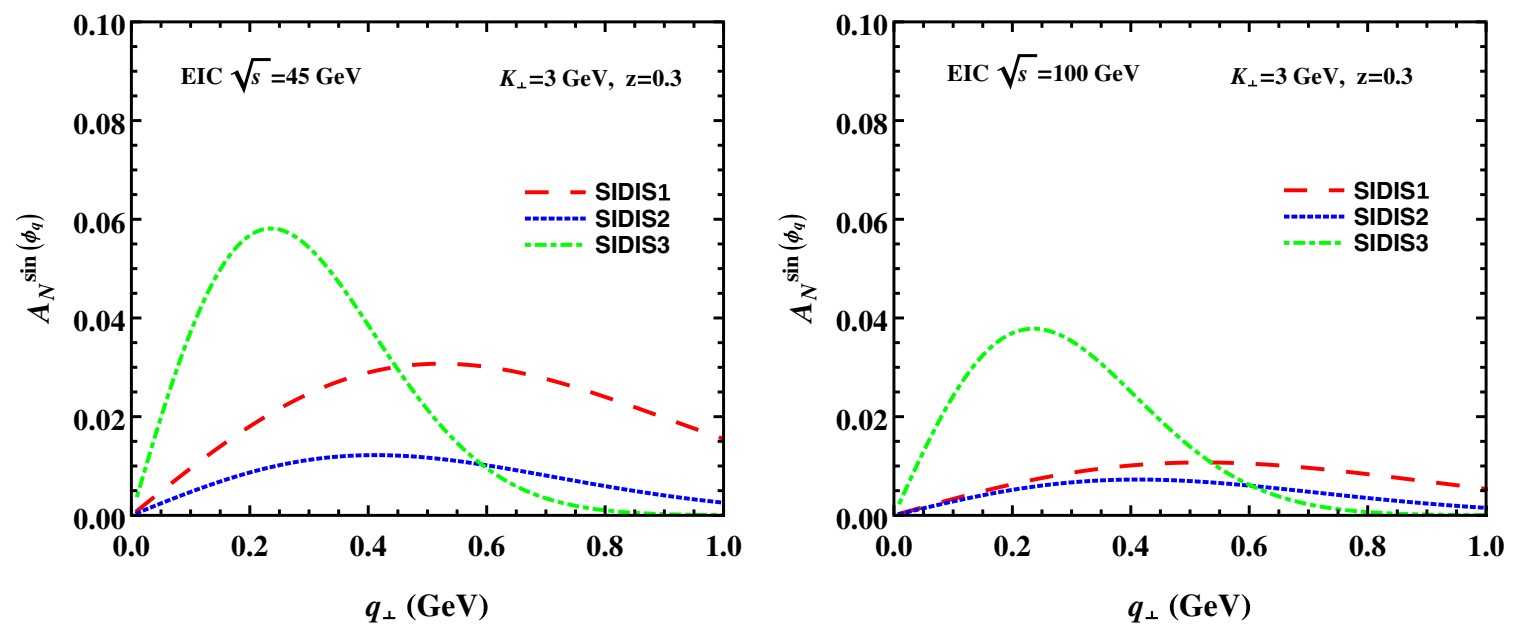

FIG. 3. The weighted Sivers asymmetry in the $e+p^{\uparrow} \rightarrow J / \psi+$ jet $+X$ process as a function of $q_{\perp}$ at EIC (a) $\sqrt{s}=45 \mathrm{GeV}$ and (b) $\sqrt{s}=$ $100 \mathrm{GeV}$ using the DGLAP evolution approach for SIDIS1, SIDIS2, and SIDIS3 GSF parametrization sets which are given in Table I.
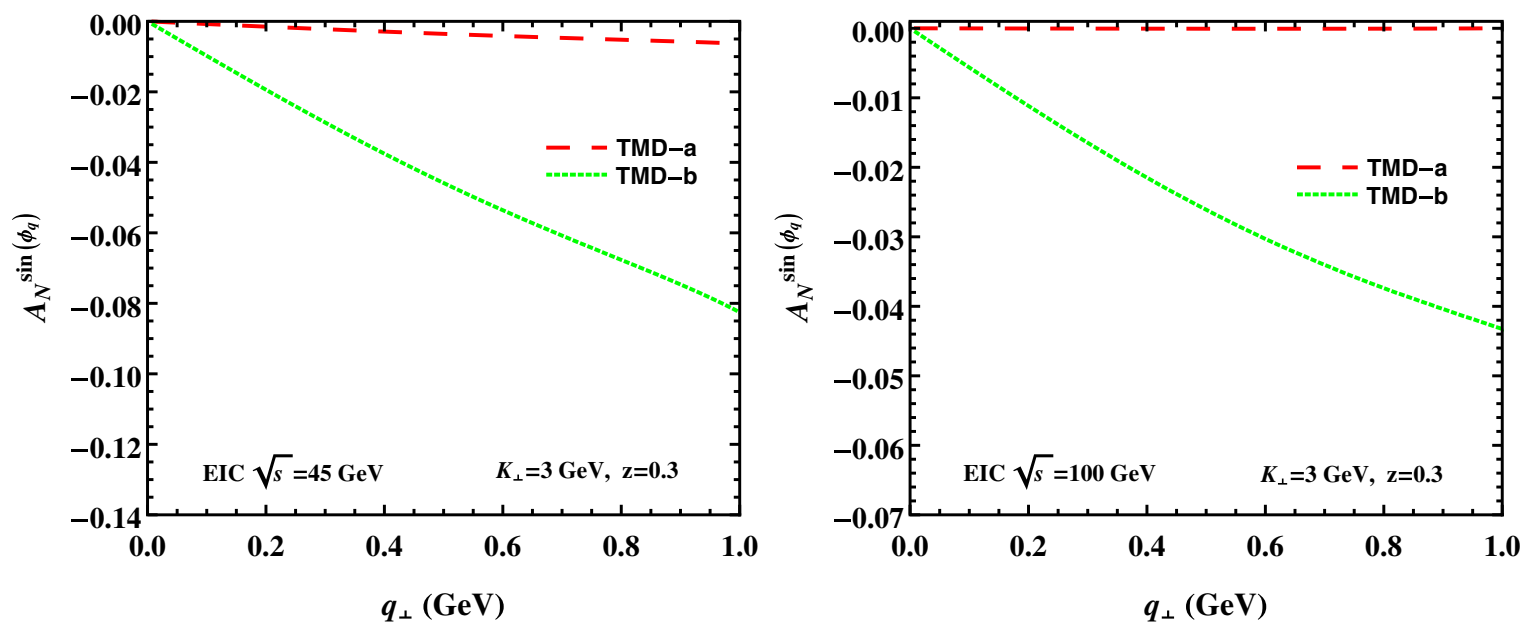

FIG. 4. The weighted Sivers asymmetry in the $e+p^{\uparrow} \rightarrow J / \psi+$ jet $+X$ process as a function of $q_{\perp}$ at EIC (a) $\sqrt{s}=45 \mathrm{GeV}$ and (b) $\sqrt{s}=100 \mathrm{GeV}$ using the TMD evolution approach for TMD-a and TMD-b GSF parametrization sets which are given in Table I. 
the kinematical region considered here. We have neglected the quark channel contribution to the numerator part of the asymmetry in Figs. 3 and 4. In the right panel of Fig. 2, the individual CS and $\mathrm{CO}$ states contribution to $A_{N}^{\mathrm{Max}}$ is shown. The ${ }^{3} S_{1}^{(1)}$ and ${ }^{1} S_{0}^{(8)}$ states contribute largely to $A_{N}^{\mathrm{Max}}$ which is independent of $\sqrt{s}$.

In the left panel of Fig. 3, the weighted Sivers asymmetry, $A_{N}^{\sin \left(\phi_{q}\right)}$, is estimated to be about $3 \%, 1 \%$, and $6 \%$, respectively, for the SIDIS1, SIDIS2, and SIDIS3 sets of GSF parameters at $\sqrt{s}=45 \mathrm{GeV}$. The $A_{N}^{\sin \left(\phi_{q}\right)}$ is reduced about $2 \%$ for $\sqrt{s}=100 \mathrm{GeV}$ as shown in the right panel of Fig. 3. In Fig. 4, negative Sivers asymmetry is shown in the TMD evolution approach. The sign of the Sivers asymmetry depends on the relative magnitude of $N_{u}$ and $N_{d}$ and these have opposite sign which can be observed in Table I. For the TMD-b parameter set, the $\mathcal{N}_{g}$ is assumed to be proportional to $\mathcal{N}_{d}$ of the $d$ quark; see Eq. (28). The value of $N_{d}$ is negative which leads to negative asymmetry. The average of $u$ and $d$ quarks $x_{a}$-dependent factor, $\mathcal{N}_{a}$, is defined for a gluon as given in Eq. (28) for the TMD-a parameter set. The magnitude of $\mathcal{N}_{d}$ is comparable but slightly dominant compared to $\mathcal{N}_{u}$ that leads to negative and small asymmetry for the TMD-a parameter set. In Fig. $4, A_{N}^{\sin \left(\phi_{q}\right)}$ is the estimated maximum of $8 \%$ and $4 \%$ at $\sqrt{s}=45$ and $100 \mathrm{GeV}$ for the TMD-b parameter set.

\section{CONCLUSION}

In this work, we gave an estimate of the Sivers asymmetry in almost back-to-back $J / \psi$ and jet photoproduction at the future EIC. We assumed TMD factorization for this process and used a generalized parton model, incorporating the intrinsic transverse momenta. The quasireal photoproduction takes place through the WeizsäkerWilliams photon distribution of the electron. We used NRQCD to calculate the $J / \psi$ production and incorporated both $\mathrm{CS}$ and $\mathrm{CO}$ contributions to the asymmetry. A major contribution comes from ${ }^{3} S_{1}^{(1)}$ and ${ }^{1} S_{0}^{(8)}$ states. We have also shown the effect of the TMD evolution on the asymmetry. In fact the Sivers asymmetry is positive without incorporating the TMD evolution, whereas it becomes negative when evolution is incorporated. We have obtained sizable Sivers asymmetry where the main contribution comes from the gluon Sivers function and the quark contribution is small. Therefore, back-to-back production of a $J / \psi$ and a jet at the future EIC is a promising tool to access the gluon Sivers function.

\section{ACKNOWLEDGMENTS}

We would like to thank Cristian Pisano and Pieter Taels for useful discussions. The work of S. R. is supported by Fondazione Sardegna under the project Quarkonium at LHC energies, CUP F71I17000160002 (University of Cagliari). A. M. would like to thank University of
Cagliari and INFN, Cagliari, Italy, for hospitality where the final stage of this work was completed.

\section{APPENDIX A: KINEMATICS}

We consider the frame in which the proton and electron are moving along $+z$ and $-z$ axes, respectively, and their four momenta are given by

$$
P=\frac{\sqrt{s}}{2}(1,0,0,1), \quad l=\frac{\sqrt{s}}{2}(1,0,0,-1) .
$$

The center-of-mass energy of the electron-proton system is $s=(P+l)^{2}$. The above four momenta in a light-cone coordinate system can be written as

$$
P^{\mu}=\sqrt{\frac{s}{2}} n_{+}^{\mu}, \quad l^{\mu}=\sqrt{\frac{s}{2}} n_{-}^{\mu},
$$

where $n_{+}$and $n_{-}$are two lightlike vectors with $n_{+} \cdot n_{-}=1$ and $n_{+}^{2}=n_{-}^{2}=0$,

$$
n_{+}^{\mu}=(1,0, \mathbf{0}), \quad n_{-}^{\mu}=(0,1, \mathbf{0}) .
$$

We assume that the quasireal photon is collinear to the electron. The quasireal photon and parton four momenta are given by

$$
\begin{gathered}
q^{\mu}=x_{\gamma} \sqrt{\frac{s}{2}} n_{-}^{\mu}, \\
p=\frac{p_{\perp a}^{2}}{2 x_{a} \sqrt{\frac{s}{2}}} n_{-}^{\mu}+x_{a} \sqrt{\frac{s}{2}} n_{+}^{\mu}+p_{\perp a}^{\mu} \approx x_{a} \sqrt{\frac{s}{2}} n_{+}^{\mu}+\boldsymbol{p}_{\perp a}^{\mu},
\end{gathered}
$$

where $x_{\gamma}=\frac{q^{-}}{l^{-}}$and $x_{a}=\frac{p^{+}}{P^{+}}$are the light-cone momentum fractions. The four momentum of the $J / \psi$ and final parton are given by

$$
\begin{gathered}
P_{\Psi}^{\mu}=z x_{\gamma} \sqrt{\frac{s}{2}} n_{-}^{\mu}+\frac{M^{2}+P_{\Psi \perp}^{2}}{2 z x_{\gamma} \sqrt{\frac{s}{2}}} n_{+}^{\mu}+\boldsymbol{P}_{\Psi \perp}^{\mu}, \\
P_{j}^{\mu}=z_{1} x_{\gamma} \sqrt{\frac{s}{2}} n_{-}^{\mu}+\frac{P_{j \perp}^{2}}{2 z_{1} x_{\gamma} \sqrt{\frac{s}{2}}} n_{+}^{\mu}+\boldsymbol{P}_{j \perp}^{\mu} .
\end{gathered}
$$

The inelastic variables are defined as $z=\frac{P \cdot P_{\Psi}}{P \cdot q}=\frac{P_{\Psi}^{-}}{q^{-}}$and $z_{1}=\frac{P \cdot P_{j}}{P \cdot q}=\frac{P_{j}^{-}}{q^{-}}$. By using the above relations, we can write down the expressions of Mandelstam variables as

$$
\begin{gathered}
\hat{s}=(q+p)^{2}=2 k \cdot q=s x_{a} x_{\gamma}, \\
\hat{t}=\left(q-P_{j}\right)^{2}=-2 q \cdot P_{j}=-\frac{P_{j \perp}^{2}}{z_{1}},
\end{gathered}
$$




$$
\begin{aligned}
\hat{u} & =\left(q-P_{\Psi}\right)^{2}=M^{2}-2 q \cdot P_{\Psi} \\
& =M^{2}-\frac{M^{2}+P_{\Psi \perp}^{2}}{z} .
\end{aligned}
$$

Here $M$ is the mass of $J / \psi$.

\section{APPENDIX B: MATRIX ELEMENTS FOR $\gamma+q(\bar{q}) \rightarrow J / \psi+q(\bar{q})$ SUBPROCESSES}

In this section the matrix elements for the $\gamma+q(\bar{q}) \rightarrow$ $J / \psi+q(\bar{q})$ channel are presented:

$$
\left|\mathcal{M}\left({ }^{3} S_{1}^{(1)}\right)\right|^{2}=0,
$$

$\left|\mathcal{M}\left({ }^{3} P_{2}^{(8)}\right)\right|^{2}=\frac{32(4 \pi)^{3} e_{c}^{2} \alpha_{s}^{2} \alpha}{45 M^{3} \hat{u}(\hat{s}+\hat{t})^{4}}\left\langle 0\left|\mathcal{O}_{8}^{J / \psi}\left({ }^{3} P_{2}\right)\right| 0\right\rangle\left[-\hat{u}^{2}\left(7 \hat{s}^{2}+12 \hat{s} \hat{t}+7 \hat{t}^{2}\right)-12 \hat{u}\left(\hat{s}^{2}+\hat{s} \hat{t}+\hat{t}^{2}\right)(\hat{s}+\hat{t})-6\left(\hat{s}^{2}+\hat{t}^{2}\right)(\hat{s}+\hat{t})^{2}\right]$.

The matrix elements for the $\gamma g \rightarrow J / \psi+g$ channel are given in our previous paper [29].

[1] D. W. Sivers, Phys. Rev. D 41, 83 (1990).

[2] M. Burkardt, Nucl. Phys. A735, 185 (2004).

[3] J.-w. Qiu and G. F. Sterman, Phys. Rev. Lett. 67, 2264 (1991).

[4] A. Airapetian et al. (HERMES Collaboration), Phys. Rev. Lett. 94, 012002 (2005).

[5] C. Adolph et al. (COMPASS Collaboration), Phys. Lett. B 717, 383 (2012).

[6] M. Anselmino, M. Boglione, U. D'Alesio, F. Murgia, and A. Prokudin, J. High Energy Phys. 04 (2017) 046.

[7] U. D’Alesio, F. Murgia, and C. Pisano, J. High Energy Phys. 09 (2015) 119.

[8] U. D'Alesio, C. Flore, F. Murgia, C. Pisano, and P. Taels, Phys. Rev. D 99, 036013 (2019).

[9] S. M. Aybat, J. C. Collins, J.-W. Qiu, and T.C. Rogers, Phys. Rev. D 85, 034043 (2012).

[10] S. M. Aybat and T. C. Rogers, Phys. Rev. D 83, 114042 (2011).

[11] J. Collins and T.C. Rogers, Phys. Rev. D 96, 054011 (2017).

[12] M. G. Echevarria, I. Scimemi, and A. Vladimirov, J. High Energy Phys. 09 (2016) 004.

[13] M. G. Echevarria, A. Idilbi, Z.-B. Kang, and I. Vitev, Phys. Rev. D 89, 074013 (2014).

[14] A. Accardi et al., Eur. Phys. J. A 52, 268 (2016).

[15] S. J. Brodsky, F. Fleuret, C. Hadjidakis, and J. P. Lansberg, Phys. Rep. 522, 239 (2013).
[16] D. Kikoa, M. G. Echevarria, C. Hadjidakis, J.-P. Lansberg, C. Lorc, L. Massacrier, C. M. Quintans, A. Signori, and B. Trzeciak, Few-Body Syst. 58, 139 (2017).

[17] B. Trzeciak, C. Da Silva, E. G. Ferreiro, C. Hadjidakis, D. Kikola, J. P. Lansberg, L. Massacrier, J. Seixas, A. Uras, and Z. Yang, Few-Body Syst. 58, 148 (2017).

[18] P. J. Mulders and J. Rodrigues, Phys. Rev. D 63, 094021 (2001).

[19] M. Burkardt, Phys. Rev. D 69, 091501 (2004).

[20] M. Anselmino, M. Boglione, U. D’Alesio, A. Kotzinian, S. Melis, F. Murgia, A. Prokudin, and C. Turk, Eur. Phys. J. A 39, 89 (2009).

[21] S. J. Brodsky, D. S. Hwang, and I. Schmidt, Nucl. Phys. B642, 344 (2002).

[22] D. Boer, P. J. Mulders, and F. Pijlman, Nucl. Phys. B667, 201 (2003).

[23] L. Adamczyk et al. (STAR Collaboration), Phys. Rev. Lett. 116, 132301 (2016).

[24] M. Aghasyan et al. (COMPASS Collaboration), Phys. Rev. Lett. 119, 112002 (2017).

[25] C. J. Bomhof and P. J. Mulders, J. High Energy Phys. 02 (2007) 029.

[26] M. G. A. Buffing, A. Mukherjee, and P. J. Mulders, Phys. Rev. D 88, 054027 (2013).

[27] R. Kishore and A. Mukherjee, Phys. Rev. D 99, 054012 (2019). 
[28] J.-P. Lansberg, C. Pisano, and M. Schlegel, Nucl. Phys. B920, 192 (2017).

[29] S. Rajesh, R. Kishore, and A. Mukherjee, Phys. Rev. D 98, 014007 (2018).

[30] A. Mukherjee and S. Rajesh, Eur. Phys. J. C 77, 854 (2017).

[31] U. D’Alesio, F. Murgia, C. Pisano, and P. Taels, Phys. Rev. D 96, 036011 (2017).

[32] A. Mukherjee and S. Rajesh, Phys. Rev. D 93, 054018 (2016).

[33] A. Mukherjee and S. Rajesh, Phys. Rev. D 95, 034039 (2017).

[34] J. Matouek (COMPASS Collaboration), J. Phys. Conf. Ser. 678, 012050 (2016).

[35] U. D’Alesio, F. Murgia, C. Pisano, and P. Taels, Phys. Rev. D 100, 094016 (2019).

[36] U. D'Alesio, C. Flore, and F. Murgia, Phys. Rev. D 95, 094002 (2017).

[37] R. M. Godbole, A. Misra, A. Mukherjee, and V. S. Rawoot, Phys. Rev. D 85, 094013 (2012).

[38] R. M. Godbole, A. Misra, A. Mukherjee, and V. S. Rawoot, Phys. Rev. D 88, 014029 (2013).

[39] M. G. Echevarria, J. High Energy Phys. 10 (2019) 144.

[40] C. E. Carlson and R. Suaya, Phys. Rev. D 14, 3115 (1976).

[41] E. L. Berger and D. L. Jones, Phys. Rev. D 23, 1521 (1981).

[42] R. Baier and R. Ruckl, Phys. Lett. 102B, 364 (1981).

[43] R. Baier and R. Ruckl, Nucl. Phys. B201, 1 (1982).

[44] E. Braaten and S. Fleming, Phys. Rev. Lett. 74, 3327 (1995).

[45] P. L. Cho and A. K. Leibovich, Phys. Rev. D 53, 150 (1996).

[46] P. L. Cho and A. K. Leibovich, Phys. Rev. D 53, 6203 (1996).

[47] G. P. Lepage, L. Magnea, C. Nakhleh, U. Magnea, and K. Hornbostel, Phys. Rev. D 46, 4052 (1992).

[48] F. Abe et al. (CDF Collaboration), Phys. Rev. Lett. 79, 572 (1997).

[49] D. Acosta et al. (CDF Collaboration), Phys. Rev. D 71, 032001 (2005).
[50] C. Adloff et al. (H1 Collaboration), Eur. Phys. J. C 25, 25 (2002).

[51] F. D. Aaron et al. (H1 Collaboration), Eur. Phys. J. C 68, 401 (2010).

[52] S. Chekanov et al. (ZEUS Collaboration), Eur. Phys. J. C 27, 173 (2003).

[53] H. Abramowicz et al. (ZEUS Collaboration), J. High Energy Phys. 02 (2013) 071.

[54] S. Frixione, M. L. Mangano, P. Nason, and G. Ridolfi, Phys. Lett. B 319, 339 (1993).

[55] D. Boer, P. J. Mulders, C. Pisano, and J. Zhou, J. High Energy Phys. 08 (2016) 001.

[56] A. Bacchetta, U. D'Alesio, M. Diehl, and C. A. Miller, Phys. Rev. D 70, 117504 (2004).

[57] A. Bacchetta, C. Bomhof, U. D'Alesio, P. J. Mulders, and F. Murgia, Phys. Rev. Lett. 99, 212002 (2007).

[58] J. Collins, Foundations of Perturbative QCD (Cambridge University Press, Cambridge, England, 2013).

[59] S. M. Aybat, J. C. Collins, J.-W. Qiu, and T. C. Rogers, Phys. Rev. D 85, 034043 (2012).

[60] X. Ji, J.-W. Qiu, W. Vogelsang, and F. Yuan, Phys. Rev. Lett. 97, 082002 (2006).

[61] C. Kouvaris, J.-W. Qiu, W. Vogelsang, and F. Yuan, Phys. Rev. D 74, 114013 (2006).

[62] D. Boer and W. Vogelsang, Phys. Rev. D 69, 094025 (2004).

[63] F. Yuan, Phys. Rev. D 78, 014024 (2008).

[64] K.-T. Chao, Y.-Q. Ma, H.-S. Shao, K. Wang, and Y.-J. Zhang, Phys. Rev. Lett. 108, 242004 (2012).

[65] Y.-d. Li and L.-s. Liu, Commun. Theor. Phys. 29, 99 (1998).

[66] M. Butenschoen and B. A. Kniehl, Phys. Rev. Lett. 104, 072001 (2010).

[67] P. Artoisenet, J. M. Campbell, F. Maltoni, and F. Tramontano, Phys. Rev. Lett. 102, 142001 (2009).

[68] A. Buckley, J. Ferrando, S. Lloyd, K. Nordstrm, B. Page, M. Rfenacht, M. Schnherr, and G. Watt, Eur. Phys. J. C 75, 132 (2015). 